\title{
The effect of a practice-based multicomponent intervention that includes health coaching on medication adherence and blood pressure control in rural primary care
}

\author{
Jia-Rong Wu PhD ${ }^{1}$ (D) | Doyle M. Cummings PharmD, FCP, FCCP ${ }^{2,3}$ | Quefeng Li PhD ${ }^{4}$ | \\ Alan Hinderliter MD ${ }^{5}$ | Hayden B. Bosworth PhD $^{6}$ | Jimmy Tillman M.Div ${ }^{7}$ | \\ Darren DeWalt MD, $\mathrm{MPH}^{8}$
}

\author{
${ }^{1}$ School of Nursing, University of North \\ Carolina, Chapel Hill, NC, USA \\ ${ }^{2}$ Department of Family Medicine, Brody \\ School of Medicine at East Carolina \\ University, Greenville, NC, USA \\ ${ }^{3}$ School of Pharmacy, University of North \\ Carolina, Chapel Hill, NC, USA \\ ${ }^{4}$ Department of Biostatistics, Gillings School \\ of Global Public Health, Chapel Hill, NC, USA \\ ${ }^{5}$ Division of Cardiology, School of Medicine, \\ Chapel Hill, NC, USA \\ ${ }^{6}$ Department of Population Health Sciences \\ at Duke University, Durham, NC, USA \\ ${ }^{7}$ Open Water Coaching and \\ Consulting, LLC, Cape Carteret, NC, USA \\ ${ }^{8}$ Department of General Internal \\ Medicine, School of Medicine, Chapel Hill, \\ NC, USA
}

\section{Correspondence}

Jia-Rong Wu, PhD, RN, School of Nursing, University of North Carolina, Chapel Hill, NC, USA.

Email: jiarongw@email.unc.edu

Funding information

National Heart Lung and Blood Institute of the National Institutes of Health, Grant/ Award Number: NHLBI 1P50HL105184
Low adherence to anti-hypertensive medications contributes to worse outcomes. The authors conducted a secondary data analysis to examine the effects of a healthcoaching intervention on medication adherence and blood pressure (BP), and to explore whether changes in medication adherence over time were associated with changes in BP longitudinally in 477 patients with hypertension. Data regarding medication adherence and BP were collected at baseline, 6, 12, 18, and 24 months. The intervention resulted in increases in medication adherence $(5.75 \rightarrow 5.94, P=.04)$ and decreases in diastolic BP $(81.6 \rightarrow 76.1 \mathrm{~mm} \mathrm{Hg}, P<.001)$ over time. The changes in medication adherence were associated with reductions in diastolic BP longitudinally $(P=.047)$. Patients with low medication adherence at baseline had significantly greater improvement in medication adherence and BP over time than those with high medication adherence. The intervention demonstrated improvements in medication adherence and diastolic BP and offers promise as a clinically applicable intervention in rural primary care.

\section{1 | INTRODUCTION}

High blood pressure (hypertension [HTN]) is a prevalent chronic condition, affecting with 1 in 3 (34\%) adult Americans 20 years and older. ${ }^{1}$ It is the most frequent chronic medical condition among adults aged 65 years and older ${ }^{2}$ and is a leading risk factor for many cardiovascular conditions, including heart attack, stroke, aneurysm, and heart failure. ${ }^{1,3}$ In the US, the estimated direct and indirect cost of HTN for 2012-2013 was $\$ 51.2$ billion and may increase to an estimated $\$ 200$ billion by 2030 . $^{1}$

From NHANES data, $17.2 \%$ of US adults are not aware that they have high blood pressure (BP). ${ }^{4}$ Moreover, $\mathrm{HTN}$ is not well-controlled, $75.7 \%$ were currently taking medication to lower their BP, but only $51.9 \%$ of those who took antihypertensive drug treatment achieved 
a BP goal of less than $140 / 90 \mathrm{~mm} \mathrm{Hg} .{ }^{4}$ From an estimate derived from a mathematical model, a $10 \%$ increase in hypertension treatment/control (ie, risk factor reduction and use of medications) would result in 14000 prevented deaths. ${ }^{5}$

Many interventions have been tested to improve BP control in patients with HTN. ${ }^{6-12}$ The findings from randomized controlled trials (RCTs) have indicated that the use of antihypertensive drug therapy is one of the most effective ways to reduce both systolic and diastolic BP. ${ }^{6}$ Medication adherence is therefore critical to achieve and sustain BP control, ${ }^{3,13}$ but barriers to optimal medication adherence are complex and multi-dimensional, ${ }^{14}$ especially for rural patients. ${ }^{15}$

Health coaching is an important intervention to provide patients with education, information, and skills needed to support a healthy lifestyle, self-manage their chronic condition(s), and prevent exacerbations or complications of chronic illness at home. This coaching can be delivered by clinicians or peers ${ }^{16}$ and has shown promising effects in improving outcomes. ${ }^{9,17-19}$ In a multicenter RCT entitled Coaching Patients on Achieving Cardiovascular Health (COACH), patients in the coaching intervention group had significantly greater reductions in total cholesterol level, BP, body weight, body mass index, dietary intake of total fat, saturated fat, and cholesterol. ${ }^{17}$ Among patients with HTN, a series of phone health-coaching intervention studies have shown that participants in the health-coaching group improved their medication adherence ${ }^{19}$ and decreased their systolic and diastolic BP compared to the control group. .,18,19 $^{-19}$

However, no studies have reported on the effectiveness of interventions that include literacy-sensitive (ie, easy-to-understand educational materials) health coaching when specifically used in populations with larger numbers of African-American hypertensive patients in rural primary care settings. Furthermore, to our understanding, no study reported whether changes in medication adherence over time were associated with changes in systolic and diastolic BP longitudinally. Therefore, the purpose of this study was to determine whether a multicomponent quality improvement intervention in rural primary care, that included an evidence-based and literacysensitive health-coaching component, was associated with improvement in self-reported medication adherence, and subsequently, with reductions in BP. Also, to determine whether changes in medication adherence over time were associated with changes in systolic and diastolic BP longitudinally in patients with uncontrolled HTN.

\section{2 | METHODS}

\section{1 | Study design}

This study was a secondary data analysis of the Heart Healthy Lenoir ( $\mathrm{HHL}$ ) hypertension Project that was designed to reduce disparities in HTN control in a rural county in NC; details of the $\mathrm{HHL}$ project were previously published. ${ }^{20,21}$ In this study, investigators focused on changes in medication adherence and associated changes in BP from a prospective, practice-based, multicomponent quality improvement intervention that included an evidence-based and literacy-sensitive health-coaching component. The prospective study was conducted in rural primary care settings and evaluated using a cohort of patients with uncontrolled HTN.

\subsection{1 | Sample and setting}

Detailed eligibility criteria, recruitment methods, data collection processes, intervention components, and rationale for this study have been published previously. ${ }^{20}$ In short, the study took place across 6 primary care practice settings that were all in a rural county (ie, non-metropolitan statistical area) in North Carolina with a large minority population. Participants who were 18 years and older, current patients from 1 of the 6 participating practices in the study, had a systolic $B P \geq 150 \mathrm{~mm} \mathrm{Hg}$ during at least one office visit in the last year from the electronic health records (EHRs) and/or medical records, and provided informed consent were enrolled in this study. A CONSORT diagram of participant enrollment as well as primary blood pressure outcomes have been provided in a prior publication. ${ }^{21}$

\subsection{2 | Health-coaching component of the intervention}

The practice-based phone health-coaching component was embedded in a larger quality improvement intervention involving strategies to systematically improve care processes for hypertension management in rural primary care settings as described in detail by Halladay and colleagues. ${ }^{20}$ The evidence-based health-coaching component of the intervention was modelled on a telephone self-management intervention used in the Take Control of Your Blood pressure (TCYB) study for uncontrolled hypertensive patients., ${ }^{9,19}$ In this study, investigators worked closely with the TCYB study team to develop a literacy-sensitive phone coaching program involving monthly telephone encounters with rural practice patients using scripted healthy lifestyle and hypertension management information (ie, stress management, sodium intake, alcohol and tobacco use, healthy eating, physical activity, patients/provider interaction, medication adherence, home BP monitoring, and weight loss) that included coachsupported motivational interviewing techniques, and goal setting strategies to promote behavioral change. Telephone encounters were led by 2 local health coaches who received extensive training in the use of the scripted health-coaching materials and who practiced these skills using role playing exercises. Details of the intervention components have been previously published. ${ }^{20}$

Each consenting patient participant received: (1) a home BP monitor and instructions on accurate home BP measurement technique and were asked to measure their BP 3 times per week, and (2) 12 monthly coaching calls that included a review of home BP measurements, medications, challenges to medication adherence, and self-determined goal setting.

After baseline assessment, participants received a letter to remind them about their upcoming scheduled call with their phone coach, along with educational materials that would be discussed 
during the call. Participants were also provided with their phone coach's contact information if they had questions or needed assistance problem-solving. During each call, the coach started by reviewing the participant's recent home $\mathrm{BP}$ readings and then assessing their medication adherence over the past month ("are you taking your medicine as prescribed?"). If any problem was identified (ie, high BP readings or poor medication adherence), the phone coach would discuss the possible reasons/barriers and help participants to solve their problem. The phone coach then followed the scripted curriculum for that encounter. At the end of each call, the phone coach helped participants to set short-term goals to work on between coaching sessions and tracked progress with their goals over time.

\subsection{Measurement}

\subsection{1 | Medication adherence}

Medication adherence, the primary outcome measure, was measured by patient self-report at baseline and at 6, 12, 18, and 24 month follow-up research visits (ie, visits in addition to their routine primary care visits and specifically intended for research data collection) using the reliable and well-validated 8-item Morisky Medication Adherence Scale (MMAS-8). ${ }^{22}$ Patients were asked to respond to 7 yes-no items and 1 item using a 5 -point Likert response to assess how often over the last 2 weeks they have adhered to their medication regimen. Scores were summed for a total score that ranged from 0-8; higher scores indicating better medication adherence. The investigators also dichotomized this variable as low medication adherence (MMAS score $<6$ ) or high medication adherence (MMAS score 6-8 that included medium adherent [ 6 to $<8$ ] and high adherent [8] in the original Morisky and colleagues ${ }^{22}$ paper). The MMAS- 8 has demonstrated acceptable internal consistency (Cronbach's $\alpha=.83$ ) and good concurrent and predictive validity, and has been used in patients with hypertension. ${ }^{22}$

\subsection{2 | Blood pressure changes}

Blood pressure data was also collected at the baseline, 6, 12, 18, and 24 months research visits. BP was measured in the sitting position 3 times, and averaged at each time point using standardized methods described in the Seventh Report of the Joint National Committee on the Prevention, Detection, Evaluation, and Treatment of High Blood Pressure. ${ }^{23}$ Changes in systolic and diastolic BP were calculated by subtracting the BP measurement at each follow-up time point from the baseline BP.

\subsection{3 | Covariates}

Patient's age, gender, race/ethnicity, annual household income, health insurance, number of comorbidities, number of oral antihypertensive medications, and medication side effect (yes/no) were obtained by patient interviews using a structured questionnaire.
Health literacy was assessed by the Short Test of Functional Health Literacy in Adults (s-TOFHLA). ${ }^{24}$ This health literacy instrument is reliable and has been validated in patients with cardiovascular-related diseases and other chronic diseases. ${ }^{25,26}$ Project staff also recorded each participant's number of coaching calls completed.

As noted in the design and outcome manuscripts by Halladay and colleagues ${ }^{20}$ and Cene and colleagues, ${ }^{21} 38 \%$ of the participants also co-participated in a lifestyle component of the intervention that aimed to improve their diet quality and increase their physical activity. To adjust for the effects of the lifestyle component of the intervention in the analysis, information regarding participation in the lifestyle intervention was also collected and used in the analysis.

\section{3 | Procedure}

Permission for the conduct of the study was obtained from the University of North Carolina Biomedical Institutional Review Board (IRB). The list of potential participants (with systolic BP $\geq 150 \mathrm{~mm} \mathrm{Hg}$ ) were identified and generated from each of the 6 primary care practices in the study. The potential participants were contacted by phone and a trained research assistant confirmed their eligibility. During the enrollment visit, participants provided informed consent and completed the baseline questionnaire (eg, s-TOFHLA) and other measures (eg, BP, height, weight) and also were given a home BP monitor and associated training for proper use. Participants received monthly health-coaching phone calls over 1 year. In addition to their usual primary care visits, follow-up research only visits occurred at $6,12,18$, and 24 months post baseline.

\section{4 | Data management and analysis}

Data analyses were performed using SPSS 24 and R-3.3.0; a significance level of .05 was used throughout. Data analysis began with a descriptive examination of all variables, including frequency distributions, percent, means, and standard deviations as appropriate to the level of measurement of the variables.

The investigators used linear mixed effects modeling to explore crude temporal changes of the intervention on medication adherence (MMAS-8 score) and BP (systolic and diastolic) over time (ie, changes over the 24 months). Further, mixed modeling was used to explore whether changes in medication adherence over time were associated with changes in systolic and diastolic BP at different follow-up time points with and without controlling for the following covariates: age, gender, race, health literacy, number of oral antihypertensive medications, medication side effect, the number of coaching sessions attended, and receiving lifestyle intervention or not, as these factors might influence medication adherence and BP. In addition, we used two-sample t-test and Fisher's exact test to compare changes of MMAS scores, systolic and diastolic BP, and percentage of patients with high medication adherence from baseline to $6,12,18$, and 24 months between low and high medication adherence groups. 


\section{3 | RESULTS}

\section{1 | Patient characteristics}

We prescreened 796 patients, 574 patients attended an enrollment visit, and 49 of these were not eligible; therefore, there were 525 participants who met the inclusion criteria and enrolled in the parent study at baseline. ${ }^{21}$ Among these 525 participants, 477 were included in this study who had at least 1 record on medication adherence and BP. The majority of the patients were female, black, and reported an annual household income of $<\$ 40000$. Participants in the high adherent group were older (58.5 vs 54.9 ), had higher MMAS scores at baseline (6.6 vs 4.3), and lower DBP (78.9 vs $84.7 \mathrm{~mm} \mathrm{Hg}$ ) compared with those in the low adherent group. In addition, 112 participants did not receive/attend any coaching calls. Comparing those with and without attended coaching calls, there were no differences in any demographic, clinical characteristics, baseline MMAS score, SBP, or DBP. Baseline demographic/clinical characteristics and comparisons between high and low adherence groups and between those with and without coaching calls are given in Table 1.

\section{2 | Medication adherence and BP changes over time}

The mean baseline medication adherence as measured by the MMAS- 8 was $5.75 \pm 1.4$. Approximately $40 \%$ of the patients had low medication adherence (MMAS-8 score <6) at baseline. In a linear mixed effects model, crude temporal changes in medication adherence increased from $5.75 \pm 1.4$ at baseline to $5.94 \pm 1.33$ at 24 months follow-up, or by .0084 per month on average $(P=.04)$. Following adjustment for covariates, patients who reported medication side effects had lower medication adherence rates $(P<.001)$ across time; black patients also had lower medication adherence rates across time $(P=.02)$. Further, no differences in the response of MMAS scores were found by age or gender. There was not a clear relationship between the number of coaching sessions attended and improvement in MMAS scores $(P=.42)$, or between receiving lifestyle intervention or not and improvement in MMAS scores $(P=.42)$. In patients with low medication adherence at baseline, the relationship between the number of coaching sessions attended and improvement in MMAS scores was not significant $(P=.58)$. The medication adherence effect size of the coaching intervention was 0.14 (small effect size) for baseline and 24 months comparisons.

The crude temporal changes in BP were as follows: systolic BP decreased from $138.6 \pm 21.8 \mathrm{~mm} \mathrm{Hg}$ at baseline to $132.7 \pm 19.5 \mathrm{~mm} \mathrm{Hg}$ at 24 months follow-up (a reduction of $5.9 \mathrm{~mm} \mathrm{Hg}$ ), or by an average of $0.22-0.25 \mathrm{~mm} \mathrm{Hg}$ per month, both before and after adjustment for covariates $(P<.001)$. Diastolic BP decreased from $81.6 \pm 12.9 \mathrm{~mm} \mathrm{Hg}$ at baseline to $76.1 \pm 14.5 \mathrm{~mm} \mathrm{Hg}$ at 24 months follow-up (a reduction of $5.5 \mathrm{~mm} \mathrm{Hg}$ ), or by an average of 0.24 $0.26 \mathrm{~mm} \mathrm{Hg}$ per month, both before and after adjustment for covariates $(P<.001)$. The effect sizes were 0.29 (small effect size) for

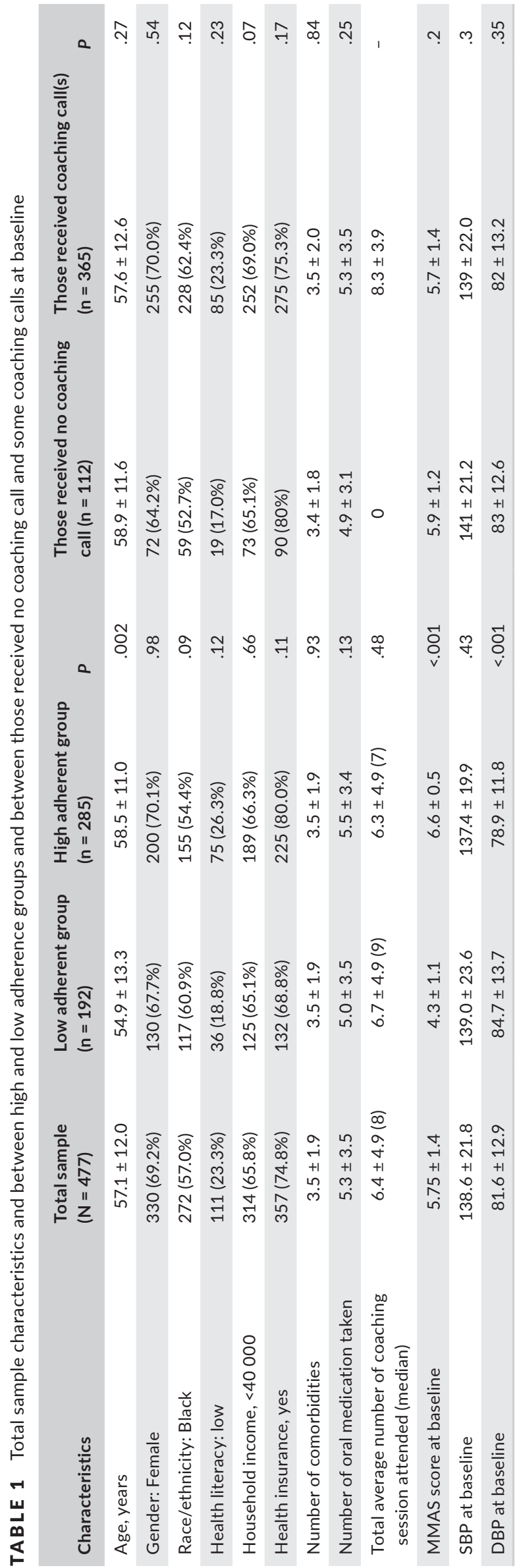


TAB LE 2 Comparisons on medication adherence and blood pressure between low and high medication adherence groups ( $\mathrm{N}=477$ )

\begin{tabular}{|c|c|c|c|c|}
\hline & 6 months-baseline & 12 months-baseline & 18 months-baseline & 24 months-baseline \\
\hline Low MA at baseline & 1.47 & 1.45 & 1.57 & 1.64 \\
\hline$P$ & $<.001$ & $<.001$ & $<.001$ & $<.001$ \\
\hline \multicolumn{5}{|c|}{ Percentage of patients with high medication adherence at different follow-up period } \\
\hline High MA at baseline & $57.9 \%$ & $68.4 \%$ & $64 \%$ & $64.9 \%$ \\
\hline$P$ & .253 & .025 & .846 & .771 \\
\hline \multicolumn{5}{|c|}{ Changes of diastolic blood pressure } \\
\hline Low MA at baseline & -7.67 & -10.18 & -9.85 & -10.47 \\
\hline Low MA at baseline & -8.21 & -11.96 & -10.25 & -9.74 \\
\hline High MA at baseline & -3.36 & -1.66 & -5.60 & -3.70 \\
\hline$P$ & .278 & .035 & .327 & .163 \\
\hline
\end{tabular}

MA, medication adherence.

systolic BP and 0.4 (medium effect size) for diastolic BP for baseline and 24 months comparisons.

\subsection{Association between medication adherence changes and BP changes over time}

In linear mixed effects models, changes in medication adherence (MMAS scores) over time were significantly associated with reductions in diastolic BP, even after adjusting for covariates. Although changes in adherence and BP and likely not linear, in the initial unadjusted model, for each 1 point increase in the MMAS score, the diastolic BP decreased by $0.053 \mathrm{~mm} \mathrm{Hg}$ more per month $(P=.07)$. In an adjusted model, if the MMAS score increased by 1 point, the diastolic BP decreased by $0.059 \mathrm{~mm} \mathrm{Hg}$ more per month $(P=.047)$. At baseline, HTN patients who had a higher MMAS score had lower diastolic BP $(P<.001)$. If a patient's MMAS score was 1 point higher than another patient's at baseline, then his/her diastolic BP was lower by $1.83 \mathrm{~mm} \mathrm{Hg}$ in the crude model and $1.89 \mathrm{~mm} \mathrm{Hg}$ in adjusted models (both $P<.001$ ).

Similarly, in linear mixed effects models evaluating changes in systolic BP, there was a trend in the expected direction but the changes did not achieve statistical significance $(P=.3$ and .42 , respectively in unadjusted and adjusted models). In the initial unadjusted model, for each 1 point increase in the Morisky score, the systolic BP decreased by $0.049 \mathrm{~mm} \mathrm{Hg}$ more per month $(P=.3)$. In an adjusted model, if the Morisky score increased by 1 point, the systolic BP decreased by $0.038 \mathrm{~mm} \mathrm{Hg}$ more per month $(P=.42)$. At baseline, HTN patients who had a higher Morisky score tended to have lower systolic BP. If a patient's Morisky score was 1 point higher than another patient's at baseline, then his/her systolic BP was lower by $0.417 \mathrm{~mm} \mathrm{Hg}$ in the crude model and $0.575 \mathrm{~mm} \mathrm{Hg}$ in adjusted models ( $P=.5$ and .36 for unadjusted and adjusted models, respectively).

\subsection{Comparisons of medication adherence scores and BP between high and low medication adherence groups}

One hundred and ninety-two patients (40.3\%) had low medication adherence and 285 patients (59.7\%) had high medication adherence at baseline (Table 2). When we compared changes of medication adherence over time between those with high and low medication adherence at baseline, MMAS scores improved by 1.5 points from baseline to 6 months in patients with low medication adherence, and the improvement was sustained at 12 months, 18 months, and 24 months follow-up. By contrast, MMAS scores decreased by 1 point from baseline to 6 months in patients with high medication adherence at baseline and stayed worse than baseline at 12, 18, and 24 months follow-up $(P<.001)$. It is important to note that among those with low medication adherence at baseline, $64 \%$ of them improved to high medication adherence at 6 months, while those with high medication adherence at baseline, only $58 \%$ of them continued to exhibit high medication adherence in follow-up; however, the difference between groups did not reach statistical significance.

Similarly, diastolic BP decreased by $8 \mathrm{~mm} \mathrm{Hg}$ from baseline to 6 months in patients with low medication adherence at baseline and stayed lower than baseline by $10 \mathrm{~mm} \mathrm{Hg}$ at 12, 18, and 24 months. By contrast, diastolic BP only decreased by $1-4 \mathrm{~mm} \mathrm{Hg}$ in patients with high medication adherence at baseline when followed from baseline to 6-24 months. The differences in diastolic BP between 
high and low medication adherence groups reached statistical significance $(P<.05)$, except at 18 months $(P=.059)$. Likewise, systolic BP decreased by $8 \mathrm{~mm} \mathrm{Hg}$ from baseline to 6 months in patients with low medication adherence and stayed lower than baseline by 10-12 mm Hg at 12, 18, and 24 months. By contrast, systolic BP only decreased by $2-6 \mathrm{~mm} \mathrm{Hg}$ in patients with high medication adherence at baseline when followed for 6-24 months. The difference between groups reached statistical significance only at 12 months $(P=.035)$; but not at 6,18 , or 24 months.

\section{DISCUSSION}

As health care delivery shifts to more quality-focused metrics, there is intensive investigation regarding optimal strategies to improve care outcomes, particularly for patients in communities with limited resources. In this study, the investigators demonstrate that a practice-based, multicomponent intervention, including monthly phone-based health coaching delivered by local health coaches, in conjunction with home BP monitoring and practice improvement activities, improves medication adherence and produces modest reduction in BP over 2 years in rural African American and white patients with a history of uncontrolled HTN. These findings are consistent with prior investigators' findings, ${ }^{8,9,11}$ but extend this work to include rural and minority patients with uncontrolled hypertension in a primary care anchored delivery model.

The coaching intervention effect on medication adherence is consistent with the prior intervention studies, including the TCYB study ${ }^{19}$ and a RCT in patients with poorly controlled diabetes, hypertension, and/or hyperlipidemia by Thom and colleagues. ${ }^{27}$ However, although the TCYB study followed participants for 24 months, only 6-month data on medication adherence were reported. ${ }^{19}$ In this study, we reported 24-month longitudinal data on medication adherence. We not only found that our coaching intervention improved medication adherence over time; most importantly, we also found that changes in medication adherence were associated with the observed reductions in systolic and diastolic BP over 24 months. Specifically, at any given time point, when medication adherence increased, the magnitude of reductions in systolic and diastolic BP increased. To our knowledge, this is the first study that has reported the relationship of changes in medication adherence over time to changes in systolic and diastolic BP at different follow-up time points in hypertensive patients, specifically in rural practices. Our finding is important as it indicates that patients with greater improvement in medication adherence will experience greater reduction in systolic and diastolic BP. These findings confirm the effectiveness of the multicomponent coaching intervention on medication adherence and $\mathrm{BP}$ in rural areas, and further emphasize the important role of medication adherence in BP control. ${ }^{27,28}$

In current medication adherence literature, it is presumed that patients with lower medication adherence at baseline have significantly greater improvement in medication adherence after intervention than those with higher medication adherence, yet the evidence is lacking.
Our study confirmed that patients with low medication adherence at baseline had significantly greater improvement in medication adherence (as indicated by increased MMAS scores), systolic BP (at 12 months), and diastolic BP (at 6, 12, and 24 months) compared with patients with high medication adherence at baseline. Our findings provide support to target those with lower medication adherence as they have more room to improve and are at higher risk of poor outcomes.

However, it is not clear why medication adherence decreased slightly from baseline to $6,12,18$, and 24 months follow-up in patients with high medication adherence at baseline. This study is a secondary data analysis. Our main criteria for including potential participants in the parent study were those with a systolic $\mathrm{BP} \geq 150 \mathrm{~mm} \mathrm{Hg}$ during at least one office visit in the last year. Therefore, we included participants with all levels of medication adherence at baseline as medication adherence decreases over time regardless of whether patients are adherent on first assessment or not. ${ }^{29}$ In a prior $\mathrm{RCT},{ }^{29}$ investigators enrolled both adherent (67\%) and non-adherent (33\%) participants in the trial. The percentage of adherent patients increased in the intervention group; yet in the control group, the percentage of adherent patients dropped from $64 \%$ at baseline to $59 \%$ at 2 months and to $36 \%$ at 9 months, indicating that even adherent patients need education and reminders to maintain/sustain their medication adherence. However, our results did demonstrate that participants with high medication adherence at baseline might need different strategies and educational content to maintain/sustain their medication adherence compared with those with low medication adherence at baseline. Therefore, interventions targeting those with high medication adherence initially need to be developed and tested in future research.

Our study has several limitations. First, this was not a RCT; instead, this is a community-based pre- and post-intervention cohort study and the potential for selection bias cannot be excluded. The investigators specifically chose a pragmatic pre- and post-intervention design, which was requested by community stakeholders in this community-based participatory research project, to facilitate study implementation in this rural community environment and also to help address a history of mistrust of academic research by some minority populations. Second, the participants in our study were from one rural county in NC; thus, these findings cannot be extrapolated to different geographic areas or other ethnic groups. Third, we did not collect clinical data that might have an impact on medication adherence and BP control, such as BP data from patients' clinic visits and whether physicians made changes in patients' anti-hypertensive medication regimen (eg, medication, dose, frequency) during the study period. In addition, medication adherence was measured mainly by self-report method. In our prior study, ${ }^{30}$ we reported that in a small subsample of 32 participants, community pharmacy fill/ refill data were available for antihypertensive medications to calculate medication possession ratio (MPR) as an additional measure of medication adherence, and we found a moderate correlation of .54 between MPR and MMAS score, indicating the reliability of using the MMAS to measure medication adherence in the rural community. Use of both objective and self-report measures may increase 
accuracy of assessment. ${ }^{31,32}$ However, the MMAS-8 is a reliable, valid, and widely used scale that is more feasible in the primary care setting to measure medication adherence and can be assessed regularly over time. Moreover, each participant received multiple phone coaching calls to improve BP control. It is hard to distinguish which components of the intervention may have produced the greatest impact. Finally, we did not find that the improvement in medication adherence was related to the number of coaching calls completed in this study, indicating intervention effects of medication adherence were not clearly linked to the dosage of the health-coaching component. Therefore, it is not clear how many coaching calls are needed to result in better medication adherence. The effect of the intervention may not be a cumulative effect requiring completion of all or most interventions; it is possible that the intervention effect may relate to the relationship developed with the health coach and the potential impact of the coach on improvement of medication adherence and subsequent reductions of $\mathrm{BP}^{33}$

\section{5 | CONCLUSIONS}

This study had important findings: (1) implementation of a multicomponent quality improvement intervention that includes a literacysensitive health-coaching component is associated with modest, but significant, improvements in medication adherence and diastolic BP; (2) changes in medication adherence over time are associated with reductions of $\mathrm{BP}$, especially diastolic $\mathrm{BP}$, among patients with a history of uncontrolled HTN; (3) patients with low medication adherence at baseline had significantly greater improvement in medication adherence, systolic BP, and diastolic BP over time compared with those with high medication adherence at baseline.

\section{6 | IMPLICATIONS FOR PRACTICE}

A multicomponent intervention that includes a phone-delivered health-coaching component is a clinically applicable intervention that offers promise in rural primary care to facilitate improvements in medication adherence that leads to reductions of systolic and diastolic BP. It is important to target patients with lower medication adherence to improve BP control.

\section{ACKNOWLEDGMENTS}

This project was supported by funding from the National Heart Lung and Blood Institute via award number NHLBI 1P50HL105184. The funding body had no role in the collection, analysis, and interpretation of study data, nor did they play a role in the writing of the manuscript or the submission of the manuscript for publication. The content is solely the responsibility of the authors and does not necessarily represent the official views of the National Heart Lung and Blood Institute, the National Institute of Nursing Research, or the National Institutes of Health. Trial registration: ClinicalTrials.gov NCT01425515.

\section{CONFLICT OF INTEREST}

All authors have no financial or other relations that could lead a conflict of interest.

\section{ORCID}

Jia-Rong Wu iD http://orcid.org/0000-0003-2294-9463

\section{REFERENCES}

1. Benjamin EJ, Blaha MJ, Chiuve SE, et al. Heart disease and stroke statistics-2017 update: a report from the American Heart Association. Circulation. 2017;135:e146-e603.

2. Moore KL, Boscardin WJ, Steinman MA, Schwartz JB. Age and sex variation in prevalence of chronic medical conditions in older residents of U.S. nursing homes. J Am Geriatr Soc. 2012;60:756-764

3. Bailey JE, Wan JY, Tang J, Ghani MA, Cushman WC. Antihypertensive medication adherence, ambulatory visits, and risk of stroke and death. J Gen Intern Med. 2010;25:495-503.

4. Nwankwo T, Yoon SS, Burt V, Gu Q. Hypertension among adults in the United States: National Health and Nutrition Examination Survey, 2011-2012. NCHS Data Brief. 2013;133:1-8.

5. Farley TA, Dalal MA, Mostashari F, Frieden TR. Deaths preventable in the U.S. by improvements in use of clinical preventive services. Am J Prev Med. 2010;38:600-609.

6. Glynn LG, Murphy AW, Smith SM, Schroeder K, Fahey T. Interventions used to improve control of blood pressure in patients with hypertension. Cochrane Database Syst Rev. 2010;3:CD005182.

7. Agarwal R, Bills JE, Hecht TJ, Light RP. Role of home blood pressure monitoring in overcoming therapeutic inertia and improving hypertension control: a systematic review and meta-analysis. Hypertension (Dallas, Tex.: 1979). 2011;57:29-38.

8. Edelman D, Dolor RJ, Coffman CJ, et al. Nurse-led behavioral management of diabetes and hypertension in community practices: a randomized trial. J Gen Intern Med. 2015;30:626-633.

9. Bosworth HB, Olsen MK, Grubber JM, et al. Two self-management interventions to improve hypertension control: a randomized trial. Ann Intern Med. 2009;151:687-695.

10. McManus RJ, Mant J, Haque MS, et al. Effect of self-monitoring and medication self-titration on systolic blood pressure in hypertensive patients at high risk of cardiovascular disease: the TASMIN-SR randomized clinical trial. JAMA. 2014;312:799-808.

11. Zullig LL, Bosworth HB. Behavioral interventions to improve hypertension control in the Veterans Affairs healthcare system. J Clin Hypertens. 2014;16:827-837.

12. Halme L, Vesalainen R, Kaaja M, Kantola I, HOme MEasuRement of Blood Pressure Study Group. Self-monitoring of blood pressure promotes achievement of blood pressure target in primary health care. Am J Hypertens. 2005;18:1415-1420.

13. Fischer MA, Stedman MR, Lii J, et al. Primary medication nonadherence: analysis of 195,930 electronic prescriptions. J Gen Intern Med. 2010;25:284-290.

14. De Geest S, Sabate E. Adherence to long-term therapies: evidence for action. Eur J Cardiovasc Nurs. 2003;2:323.

15. Wroth TH, Pathman DE. Primary medication adherence in a rural population: the role of the patient-physician relationship and satisfaction with care. J Am Board Fam Med. 2006;19:478-486.

16. Huffman M. Health coaching: a new and exciting technique to enhance patient self-management and improve outcomes. Home Healthc Nurse. 2007;25:271-274; quiz 275-276. 
17. Vale MJ, Jelinek MV, Best JD, et al. Coaching patients On Achieving Cardiovascular Health (COACH): a multicenter randomized trial in patients with coronary heart disease. Arch Intern Med. 2003;163:2775-2783.

18. Margolius D, Bodenheimer T, Bennett $\mathrm{H}$, et al. Health coaching to improve hypertension treatment in a low-income, minority population. Ann Fam Med. 2012;10:199-205.

19. Bosworth HB, Olsen MK, Neary A, et al. Take Control of Your Blood Pressure (TCYB) study: a multifactorial tailored behavioral and educational intervention for achieving blood pressure control. Patient Educ Couns. 2008;70:338-347.

20. Halladay JR, Donahue KE, Hinderliter AL, et al. The heart healthy lenoir project - an intervention to reduce disparities in hypertension control: study protocol. BMC Health Serv Res. 2013; 13:441.

21. Cene CW, Halladay JR, Gizlice Z, et al. A multicomponent quality improvement intervention to improve blood pressure and reduce racial disparities in rural primary care practices. J Clin Hypertens (Greenwich). 2017;19:351-360.

22. Morisky DE, Ang A, Krousel-Wood M, Ward HJ. Predictive validity of a medication adherence measure in an outpatient setting. J Clin Hypertens (Greenwich). 2008;10:348-354.

23. Chobanian AV, Bakris GL, Black HR, et al. The seventh report of the joint national committee on prevention, detection, evaluation, and treatment of high blood pressure: the JNC 7 report. JAMA 2003;289:2560-2572.

24. Gazmararian JA, Baker DW, Williams MV, et al. Health literacy among medicare enrollees in a managed care organization. JAMA. 1999;281:545-551.

25. Gazmararian JA, Kripalani S, Miller MJ, Echt KV, Ren J, Rask K. Factors associated with medication refill adherence in cardiovascular-related diseases: a focus on health literacy. J Gen Intern Med. 2006;21:1215-1221.

26. Kalichman SC, Pope H, White D, et al. Association between health literacy and HIV treatment adherence: further evidence from objectively measured medication adherence. J Int Assoc Physicians AIDS Care (Chic). 2008;7:317-323.

27. Thom DH, Willard-Grace R, Hessler D, et al. The impact of health coaching on medication adherence in patients with poorly controlled diabetes, hypertension, and/or hyperlipidemia: a randomized controlled trial. J Am Board Fam Med. 2015;28:38-45.

28. Wertz D, Hou L, DeVries A, et al. Clinical and economic outcomes of the Cincinnati Pharmacy Coaching Program for diabetes and hypertension. Manag Care. 2012;21:44-54

29. Wu JR, Corley DJ, Lennie TA, Moser DK. Effect of a medicationtaking behavior feedback theory-based intervention on outcomes in patients with heart failure. J Cardiac Fail. 2012;18:1-9.

30. Cummings DM, Wu JR, Cene C, et al. Perceived social standing, medication nonadherence, and systolic blood pressure in the Rural South. J Rural Health. 2015;32:156-163.

31. Cassidy CM, Rabinovitch M, Schmitz N, Joober R, Malla A. A comparison study of multiple measures of adherence to antipsychotic medication in first-episode psychosis. J Clin Psychopharmacol. 2010;30:64-67.

32. Liu H, Golin CE, Miller LG, et al. A comparison study of multiple measures of adherence to HIV protease inhibitors. Ann Intern Med. 2001;134:968-977.

33. Andre Christie-Mizell C, Blount SA, Laster Pirtle WN, Dagadu HE, Leslie ET, Vielehr PS. Psychiatric medication, African Americans and the Paradox of Mistrust. J Natl Med Assoc. 2015;107:51-59.

How to cite this article: Wu J-R, Cummings DM, Li Q, et al. The effect of a practice-based multicomponent intervention that includes health coaching on medication adherence and blood pressure control in rural primary care. J Clin Hypertens. 2018;20:757-764. https://doi.org/10.1111/jch.13265 